\title{
The novel long noncoding RNA RPI I-357HI4.I7 acts as an oncogene by promoting cell proliferation and invasion in diffuse-type gastric cancer
}

This article was published in the following Dove Press journal:

OncoTargets and Therapy

18 May 2017

Number of times this article has been viewed

\author{
Biao Yangl,* \\ Tianhang Luol,* \\ Meijing Zhang ${ }^{2, *}$ \\ Zhengmao Lu' \\ Xuchao Xue' \\ Guoen Fang' \\ 'Department of General Surgery, \\ ${ }^{2}$ Department of Oncology, Changhai \\ Hospital, The Second Military Medical \\ University, Shanghai, People's Republic \\ of China \\ *These authors contributed equally \\ to this work
}

\begin{abstract}
Current evidence indicates that long noncoding RNAs (lncRNAs) play pivotal roles in human cancers. The present study aims to assess differentially expressed lncRNAs related to diffuse-type gastric carcinoma (DGC). Next-generation RNA sequencing was carried out to detect aberrantly expressed lncRNAs in DGC. Real-time polymerase chain reaction (RT-PCR) was performed to evaluate RP11-357H14.17 gene expression levels in DGC cell lines/tissues comparatively with normal gastric epithelial cell lines and adjacent normal tissues. The associations of RP11-357H14.17 expression levels with the clinicopathological features were also analyzed. The regulatory effects of RP11-357H14.17 on the biological behaviors of DGC cells were evaluated by MTT, colony formation assays, flow cytometry for apoptosis, wound healing assay, and transwell migration and invasion assays. RP11-357H14.17 expression was remarkably increased in DGC tissues and cell lines compared with normal gastric epithelial cells and adjacent normal tissues. High levels of RP11-357H14.17 were associated with increased tumor size, deeper depth of invasion, lymphatic metastasis, and advanced pathological stage. Further experiments demonstrated that the DGC cells MGC-803 transfected with si-RP11-357H14.17 showed reduced cell proliferation, migration, invasion, enhanced G1 phase arrest and cell apoptosis. These findings suggest that the novel lncRNA RP11-357H14.17 is associated with poor prognosis, and may serve as a potential prognostic biomarker and target for new antineoplastic therapies in human DGC.
\end{abstract}

Keywords: long noncoding RNA, RP11-357H14.17, diffuse-type gastric cancer, proliferation, invasion, metastasis

\section{Introduction}

Gastric cancer (GC) is the fourth most common malignancy and second leading cause of cancer death worldwide, especially in East Asia. ${ }^{1}$ Diffuse-type gastric carcinoma (DGC) is one of the major histological types (diffuse and intestinal types) according to the classification scale originally described by Lauren in 1965. 2,3 Diffuse-type carcinomas are poorly differentiated and composed of solitary or poorly cohesive tumor cells in the absence of gland formation. Despite recent advances in surgical techniques and the medical treatment of DGC, the prognosis of patients with DGC remains relatively poor, in the absence of reliable early diagnostic markers and therapeutic targets for the suppression of tumor metastasis. ${ }^{4-6}$ The 5 -year survival rate remains low. ${ }^{7-9}$ DGC has poorer prognosis, and occurs more frequently in younger individuals with GC. ${ }^{10,11}$ Previous studies have reported many oncogenes and tumor suppressor genes closely associated with DGC, but the highly complex molecular mechanisms underlying invasion and metastasis remain obscure. ${ }^{12}$ Therefore,
Correspondence: Guoen Fang Department of General Surgery, Changhai Hospital, No 168 Changhai Road, Yangpu District, Shanghai 200433, People's Republic of China

Tel +86 2l 31I6 I589

Email fangguoen@126.com 
identifying reliable biomarkers associated with early DGC diagnosis, effective therapy, and prognosis evaluation is of great clinical relevance.

Different from conventional coding genes, long noncoding RNAs (lncRNAs) are a class of RNA transcripts longer than 200 nucleotides, with no or limited protein-coding potential. ${ }^{13}$ Emerging evidence demonstrates that lncRNAs are closely involved in multiple biological processes, including stem cell pluripotency, cell differentiation, cell growth, cell apoptosis, and cancer metastasis. ${ }^{14-16}$ Dysregulation of lncRNAs has been reported in different cancers, including DGC. ${ }^{17,18}$ However, the overall pathophysiological contributions of lncRNAs to DGC remain largely unknown. To explore the function and mechanism of lncRNAs in DGC, we performed next-generation sequencing of 6 clinical DGC samples, and found that RP11-357H14.17 was upregulated in DGC tissues compared with adjacent normal tissues. RP11-357H14.17 was mapped to 17q21.32 with a transcript length of 11,101 bp. However, the role of RP11-357H14.17 in DGC has not been reported, and its expression and function in DGC are poorly understood.

In this research, RP11-357H14.17 expression levels in DGC issues and cell lines were evaluated by real-time polymerase chain reaction (RT-PCR). We also evaluated the associations of RP11-357H14.17 levels with clinicopathological characteristics. Moreover, its role in DGC cell proliferation and metastasis was also researched. Our findings may help further understand the role of RP11-357H14.17 as a regulator of DGC pathogenesis, which may serve as a candidate target for new diagnostics and therapies.

\section{Material and methods}

\section{Patients and clinical specimens}

Fresh primary DGC tumor tissues and matched normal adjacent tissues were collected from 48 pathologically confirmed DGC patients in Changhai Hospital (Shanghai, People's Republic of China) between May 2015 and May 2016. A total of 6 cases were used for sequencing; RP11357H14.17 expression was evaluated in 42 cases. Patients with two or more different malignancies were excluded. No patients had received preoperative radiotherapy or chemotherapy. The patient characteristics are shown in Table 1. Samples were frozen immediately in liquid nitrogen and stored at $-80^{\circ} \mathrm{C}$ until analysis. The ethics committee of ChangHai Hospital affiliated to the Second Military Medical University approved this study; written informed consent was obtained from all patients (Registration number: ChiCTR-CDC-15007379) for this study.
Table I Correlation between RPII-357HI4.I7 expression and different clinicopathological features in patients with diffuse-type gastric cancer

\begin{tabular}{|c|c|c|c|}
\hline Characteristics & $\begin{array}{l}\text { High RP I I- } \\
357 \mathrm{HI} 4.17 \\
\text { expression (\%) }\end{array}$ & $\begin{array}{l}\text { Low RPII- } \\
357 \text { H } 4.17 \\
\text { expression (\%) }\end{array}$ & $P$-value \\
\hline Age (years) & & & 0.203 \\
\hline$\geq 60$ & I5 (57.7) & II (42.3) & \\
\hline$<60$ & $6(37.5)$ & $10(62.5)$ & \\
\hline Sex & & & 0.476 \\
\hline Male & $13(58.3)$ & II (4I.7) & \\
\hline Female & $8(47.1)$ & $10(52.9)$ & \\
\hline Tumor size, cm & & & $0.029 *$ \\
\hline$\geq 5$ & $15(65.2)$ & $8(34.8)$ & \\
\hline$<5$ & $6(31.6)$ & $13(68.4)$ & \\
\hline Invasion depth & & & $0.040^{*}$ \\
\hline $\mathrm{TI}, \mathrm{T} 2$ & $3(25.0)$ & $9(75.0)$ & \\
\hline $\mathrm{T} 3, \mathrm{~T} 4$ & $18(60.0)$ & $12(40.0)$ & \\
\hline TNM stage & & & $0.027^{*}$ \\
\hline $\mathrm{I} / \mathrm{II}$ & $5(29.4)$ & $12(70.6)$ & \\
\hline III & $16(64.0)$ & $9(36.0)$ & \\
\hline Lymphatic metastasis & & & $0.006^{*}$ \\
\hline Positive & $19(63.3)$ & II (36.7) & \\
\hline Negative & $2(16.7)$ & $10(83.3)$ & \\
\hline
\end{tabular}

Note: $* P<0.05$.

Abbreviation: TNM, tumor, node, metastasis.

\section{Cell lines and culture}

Human GC cell lines (AGS, SGC-7901, MGC-803, and MKN-45) and the human normal gastric epithelial cell line GES-1 were obtained from the Institute of Biochemistry and Cell Biology of the Chinese Academy of Sciences (Shanghai, People's Republic of China). They were maintained in Roswell Park Memorial Institute 1640 medium supplemented with $10 \%$ fetal bovine serum (FBS), $100 \mathrm{U} / \mathrm{mL}$ of penicillin, and $100 \mu \mathrm{g} / \mathrm{mL}$ streptomycin sulfate. Cultures were incubated in a humidified atmosphere containing $5 \% \mathrm{CO}_{2}$ at $37^{\circ} \mathrm{C}$.

\section{Whole transcriptome library preparation and sequencing}

Total RNA from 6 paired DGC and adjacent normal tissue samples was isolated and quality controlled. The preparation of whole transcriptome libraries and deep sequencing were performed by Novogene Bioinformatics Technology Cooperation (Shanghai, People's Republic of China). Ribosomal RNA was removed and strand-specific sequencing libraries were generated following the manufacturer's instructions. RNA-Seq was performed on an Illumina; HiSeq 2000 platform (San Diego, CA, USA), and 100 bp paired-end reads were generated according to the Illumina's protocol.

Lentivirus infection and construction of stable cell lines with RP11-357H14.17 knockdown lncRNA RP11-357H14.17 short hairpin RNA (LV-lncRNA-RNAi 
(52661-1), si-RP11-357H14.17, 5'-GGCCAGGAAGTCCCAGAAATA-3') and non-targeting shRNA (si-NC, 5'-TTCTCCGAACGTGTCACGT-3') were purchased from GenePharma (Shanghai, People's Republic of China). The DGC MGC-803 cells were cultured in 6-well plates at optimal density until transfection, using Lipofectamine 2000 (Thermo Fisher Scientific, Waltham, MA, USA) according to the manufacturer's instructions. The cells were harvested for further assays after $48 \mathrm{~h}$ for quantitative RT-PCR (qRTPCR) or Western blot analyses.

\section{RNA preparation and quantitative RT-PCR}

Total RNA was extracted from tissues or cultured cells using TRIzol reagent (Thermo Fisher Scientific). For qRT-PCR, $1 \mu \mathrm{g}$ RNA was reverse transcribed into cDNA with Reverse Transcription Kit (Takara, Dalian, People's Republic of China). Real-time PCR was performed with SYBR Premix ExTaq II Kit (Takara). Data were normalized to glyceraldehyde 3-phosphate dehydrogenase levels (5'-TGACTTCAACAGCGACACCCA-3' (forward) and 5'-CACCCTGTTGCTGTAGCCAAA-3' (reverse)). The PCR primers for RP11-357H14.17 were 5'-GAACTCGGCTTCTGGCACCTC-3' (forward) and 5'-CCCCACTCCCTTTCTTCCTTG-3' (reverse). The qRT-PCR assays and data collection were performed on ABI 7500, and relative RP11-357H14.17 expression was assessed by the $2^{-\Delta \mathrm{Ct}}$ method, and converted to fold changes using the $2^{-\Delta \Delta \mathrm{Ct}}$ method.

\section{MTT assay}

Cell proliferation was analyzed by MTT assay. Briefly, $1 \times 10^{3}$ cells were seeded into 96-well plates, and incubated for 1, 2, 3,4 , and 5 days. Then, $20 \mu \mathrm{L}$ of MTT $(5 \mathrm{mg} / \mathrm{mL}$ ) (SigmaAldrich, St Louis, MO, USA) was added into each well, and incubated for another $4 \mathrm{~h}$. Then, the supernatants were removed and $150 \mu \mathrm{L}$ of dimethyl sulfoxide (Sigma-Aldrich) was added to solubilize the formazan crystals. Absorbance (optical density) was measured at $490 \mathrm{~nm}$ on a microplate reader (Molecular Devices, Sunnyvale, CA, USA). Triplicate experiments were independently repeated three times.

\section{Colony formation assay}

For the colony formation assay, cells were placed in 6-well plates and maintained in media containing $10 \%$ FBS. The medium was replaced every 4 days; after 14 days, the cells were fixed with methanol and stained with $0.1 \%$ crystal violet (Sigma-Aldrich). Visible colonies were then counted.

\section{Wound healing assay}

A total of $3 \times 10^{5}$ cells were seeded in 6 -well plates. The next day, once cultures reached $85 \%$ confluence, the cell monolayer was scratched with a sterile plastic tip and washed with culture medium. Cells were then cultured for $48 \mathrm{~h}$ with medium containing $1 \%$ FBS. At different time points, images of the plates were acquired under a microscope (IX71; Olympus, Tokyo, Japan). The distance between the two edges of the scratch was measured using the Digimizer software system (MedCalc Software, Ostend, Belgium). The assay was independently repeated three times.

\section{Cell migration and invasion assays}

For the migration assay, $5 \times 10^{4}$ cells in serum-free media were placed into the upper chamber of an insert $(8-\mu \mathrm{m}$ pore size; Millipore). In invasion assays, $1 \times 10^{5}$ cells in serum-free medium were placed into the upper chamber of an insert coated with Matrigel (Sigma-Aldrich). Culture medium containing 10\% FBS was added to the lower chamber. After incubation for $24 \mathrm{~h}$, the cells remaining on the upper membrane were removed with cotton wool. Those that had migrated or passed through the membrane were stained with $0.1 \%$ crystal violet in methanol, imaged, and counted using an IX71 inverted microscope (Olympus). Experiments were independently repeated three times.

\section{Flow cytometric analysis of the cell cycle and apoptosis}

Cells were cultured in 6-well plates for $48 \mathrm{~h}$, washed with ice-cold phosphate-buffered saline (PBS), and fixed in cold $70 \%$ ethanol overnight at $-20^{\circ} \mathrm{C}$. The cells were then washed and stained with propidium iodide (PI; Beyotime, Shanghai, People's Republic of China) containing $0.25 \mathrm{mg} / \mathrm{mL}$ of RNase A. After incubation in the dark at $4^{\circ} \mathrm{C}$ for $15 \mathrm{~min}$, the cells were analyzed by flow cytometry (FACScan; BD Biosciences, San Jose, CA, USA) using the MODFIT software (Verity Software House, Topsham, ME, USA). The percentages of cells in various cycle phases (G1, $\mathrm{S}$, and $\mathrm{G} 2 / \mathrm{M}$ phases) were recorded and compared.

Apoptosis was examined using an Annexin-V fluorescein isothiocyanate (FITC)/PI dual staining kit (KeyGen, Nanjing, People's Republic of China). Briefly, MGC-803 cells were treated with lncRNA-RP11-357H14.17 or control siRNA for $48 \mathrm{~h}$ and washed three times in cold PBS. The cells were then stained with Annexin V-FITC and PI in $500 \mu \mathrm{L}$ of binding buffer, in the dark at room temperature for $15 \mathrm{~min}$, and assessed by flow cytometry. 


\section{Statistical analysis}

All experiments were independently performed at least three times. Statistical analyses were performed with the SPSS 20.0 software (IBM Corp., Armonk, NY, USA). Enumeration data were presented as percentage (\%), and compared by Chi-squared $\left(\chi^{2}\right)$ test. Values were mean \pm standard deviation; two groups were compared by paired samples $t$-test, and multiple groups by one-way analysis of variance. Non-normally distributed data were presented as median (interquartile range), and compared with the Wilcoxon test. Statistical differences were set at $* P<0.05, * * P<0.01$. $P<0.05$ was considered statistically significant.

\section{Results}

\section{LncRNA expression profiles in diffuse- type GC and adjacent normal tissues}

First, next-generation sequencing was used to analyze differentially expressed lncRNAs in 6 paired DGC and adjacent normal tissues. The differentially expressed lncRNAs were filtered by the criteria of fold change $>2$ and $P<0.05$ between cancer and adjacent normal tissues. There were 73 up-regulated and 39 down-regulated lncRNAs in DGC samples compared with adjacent normal specimens, including 30 antisense lncRNAs, 11 intronic lncRNAs, and 71 intergenic lncRNAs. Hierarchical clustering analysis on most significantly dysregulated cancer-related lncRNAs is summarized in Figure 1. The lncRNAs showing the highest absolute expression levels in DGC tissues (fold change $>8$ ) were further assessed. This filtering process yielded $3 \mathrm{lncR}$ NAs (XIST, PVT1 and RP11-357H14.17). Notably, the lncRNAs XIST and PVT1 were shown to be overexpressed in GC. ${ }^{19,20}$ Our filtering approach was validated by the identification of XIST and PVT1. Therefore, we selected the intergenic lncRNA RP11-357H14.17 for further studies.

\section{RPI I-357HI4.I7 expression and clinicopathological factors in DGC}

To verify the involvement of RP11-357H14.17 in DGC progression, we detected RP11-357H14.17 expression levels in 42 paired DGC and adjacent normal tissues by qRT-PCR. As shown in Figure 2A-C, RP11-357H14.17 amounts were remarkably up-regulated in DGC tissues compared with adjacent normal tissues $(P<0.05)$. Compared with the normal gastric epithelial cell line, GES-1, RP11-357H14.17 expression was increased in the GC cells MGC-803 ( $P<0.0001)$, MKN-45 ( $P=0.0094)$, AGS $(P=0.0079)$, and SGC-7901 $(P=0.0009)$, especially MGC-803 cells, a DGC cell line (Figure 2D).

We further analyzed the associations of RP11-357H14.17 expression with clinicopathological features in patients with DGC. According to median RP11-357H14.17 levels, we divided the samples into high (above median value, $\mathrm{n}=21$ ) and low (below median value, $n=21$ ) RP11-357H14.17 expression groups. Chi-square test was then performed to evaluate the clinicopathological features between the two

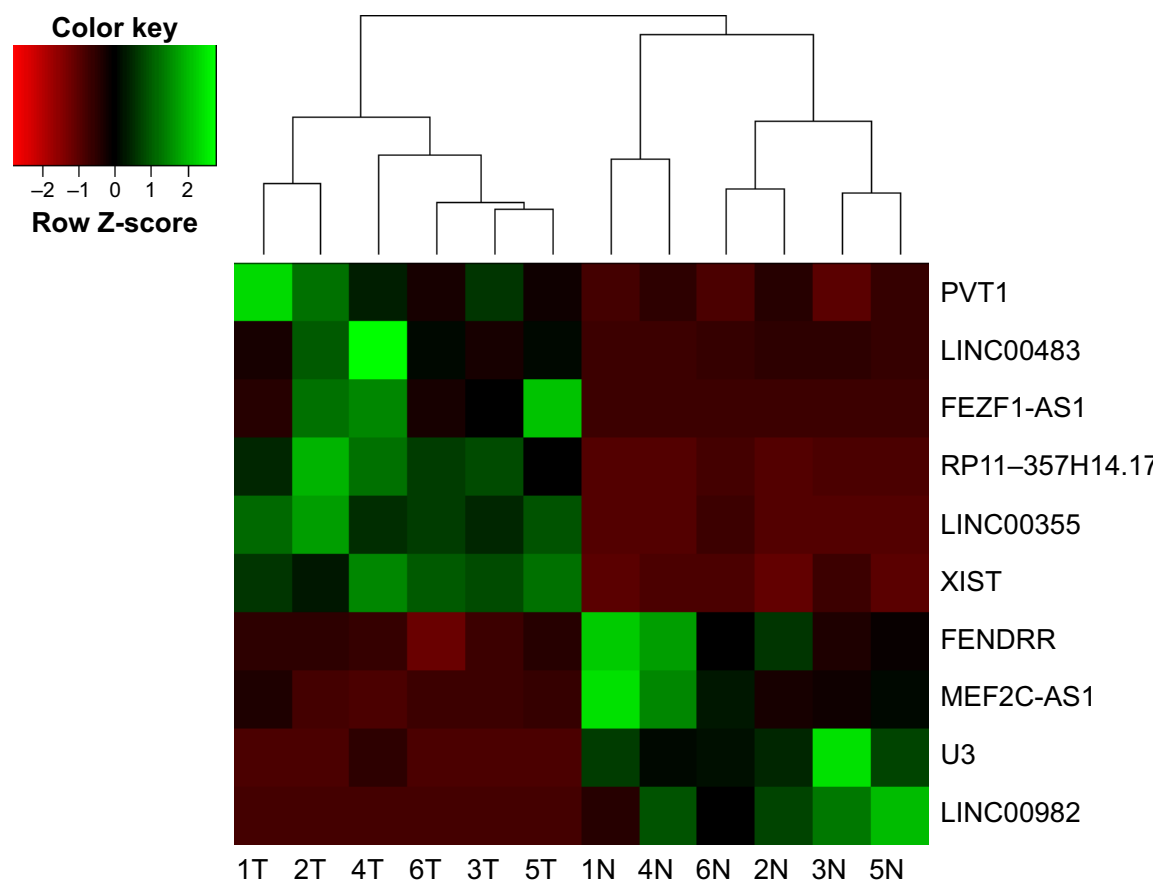

Figure I Hierarchical clustering analysis on the most significantly dysregulated cancer-related IncRNAs.

Notes: IT, 2T, 3T, 4T, 5T, 6T and IN, 2N, 3N, 4N, 5N, 6N represented cancerous and paired non-cancerous tissues of samples DGCI, DGC2, DGC3, DGC4, DGC5 and DGC6, respectively. 


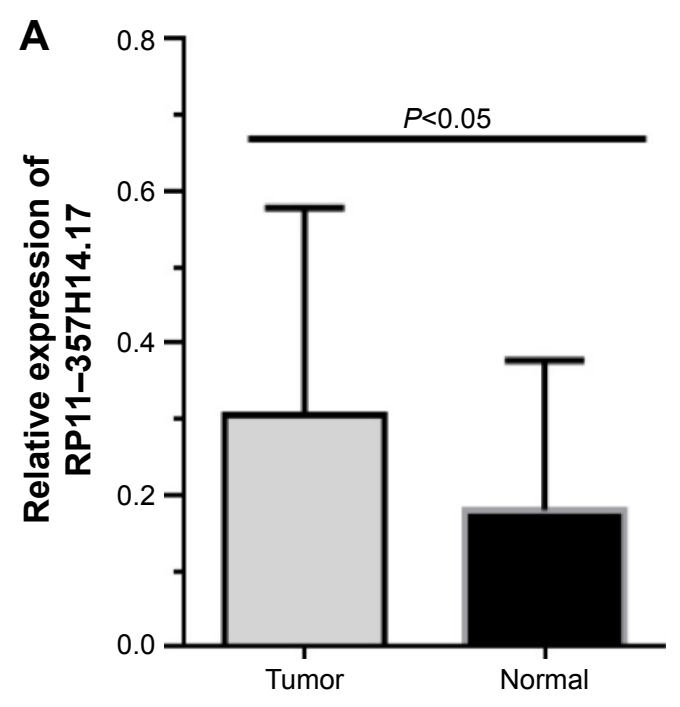

B
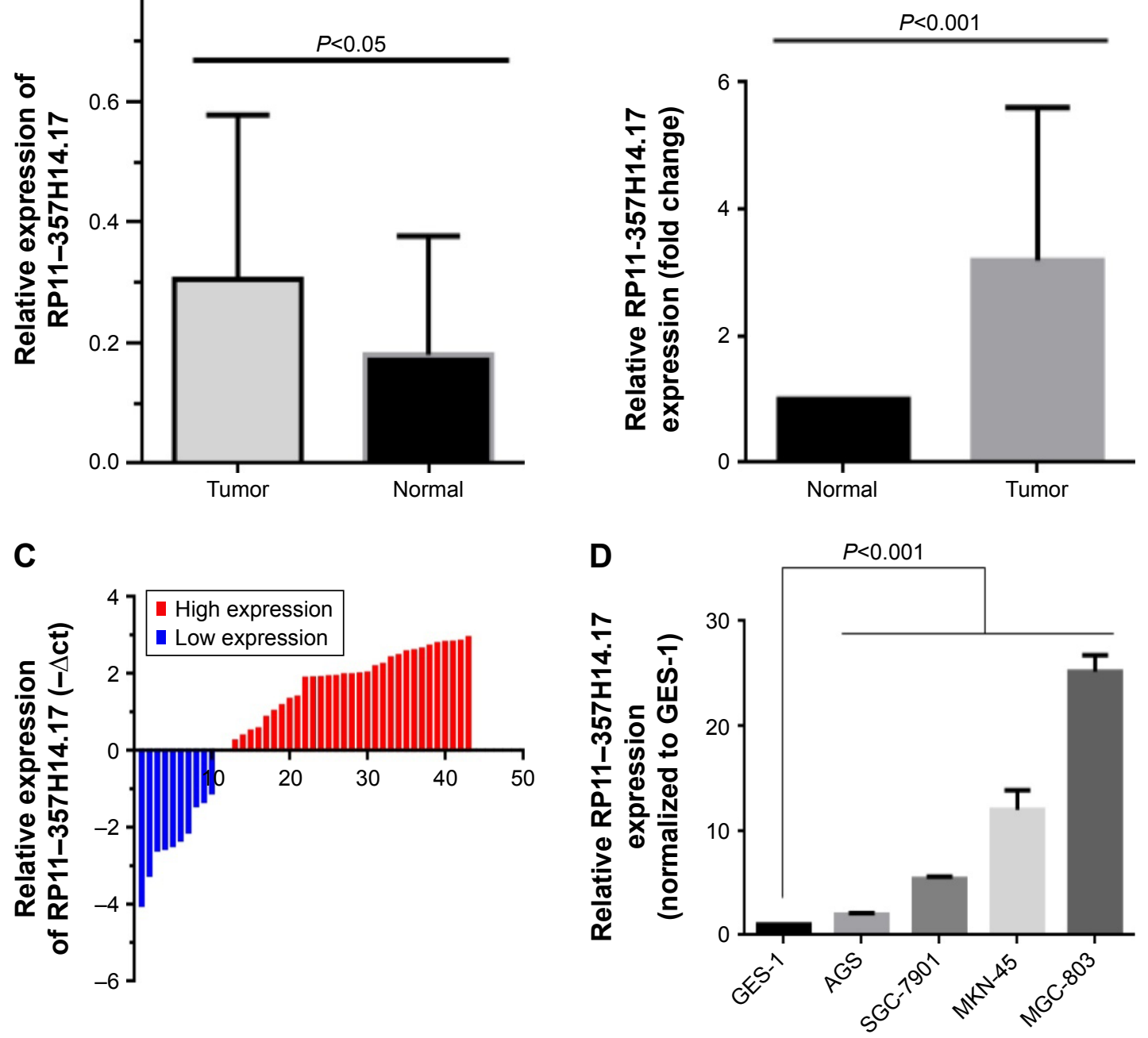

Figure 2 Relative RPI I-357HI4.I7 expression levels in diffuse-type gastric cancer tissues and cell lines.

Notes: (A) Relative expression of RPII-357HI4.I7 in 42 pairs of DGC tissues and adjacent normal tissues by qRT-PCR analysis. RPI I-357HI4.I7 levels were calculated by the $2^{-\Delta c t}$ method and normalized to glyceraldehyde 3-phosphate dehydrogenase. (B) The fold change of RPII-357HI4.I7 in DGC relative to the adjacent normal tissues in 42 DGC patients. (C) qRT-PCR detection in 42 pairs of fresh tissues showed RPII-357HI4.17 was highly expressed in DGC tissues. (D) Increased RPII-357HI4.I7 expression in $4 \mathrm{GC}$ cell lines compared with normal gastric epithelial cell line GES-I.

Abbreviations: DGC, diffuse-type gastric cancer; GC, gastric cancer; qRT-PCR, quantitative real-time polymerase chain reaction.

groups. As shown in Table 1, increased RP11-357H14.17 expression was significantly associated with larger tumor size $(P=0.029)$, deeper depth of invasion $(P=0.040)$, more advanced pathological stage $(P=0.027)$ and lymphatic metastasis $(P=0.006)$. However, RP11-357H14.17 levels were not associated with other clinicopathological features, such as age $(P=0.203)$ and sex $(P=0.476)$ in DGC.

\section{RPII-357HI4.I7 depletion inhibits proliferation and cell cycle progression, and promotes apoptosis in diffuse-type GC cells}

To assess the biological functions of RP11-357H14.17 in DGC, we designed si-RP11-357H14.17 and transfected into the MGC-803 cell line. RP11-357H14.17 expression was overtly downregulated $(P<0.005)$ in si-RP11-357H14.17 transfected cells (Figure 3A). MTT assay indicated that RP11-357H14.17 knockdown significantly inhibited cell proliferation compared with control cells after $72 \mathrm{~h}$ (Figure 3B). However, in colony formation assays, clonogenic survival was not significantly decreased following inhibition of RP11-357H14.17 in the MGC-803 cell line (Figure 3C), probably due to the short cycle. Furthermore, flow cytometry revealed that RP11-357H14.17 silencing promoted G1 phase arrest in MGC-803 cells (Figure 3D). Moreover, RP11-357H14.17 downregulation obviously promoted apoptosis compared with the si-NC group (Figure 3E). 
A

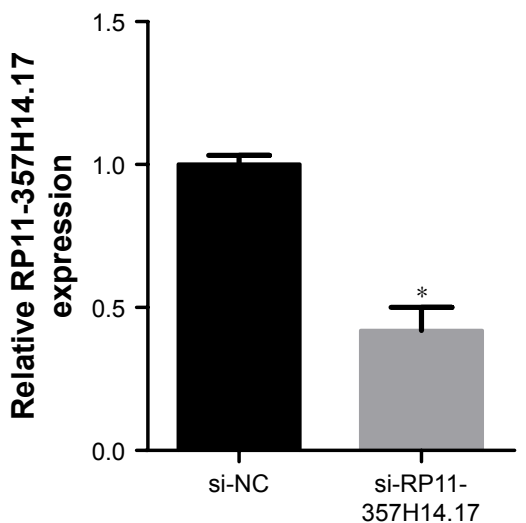

B

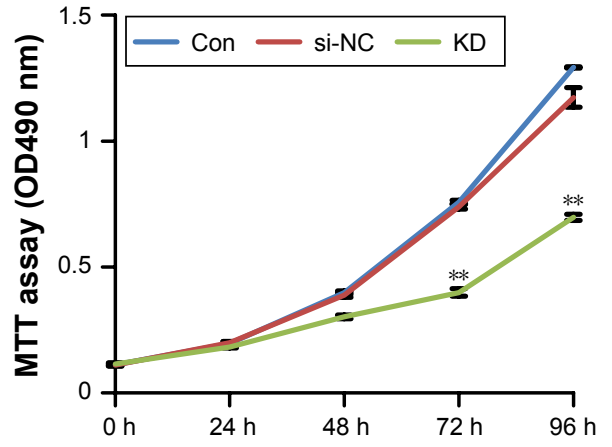

D

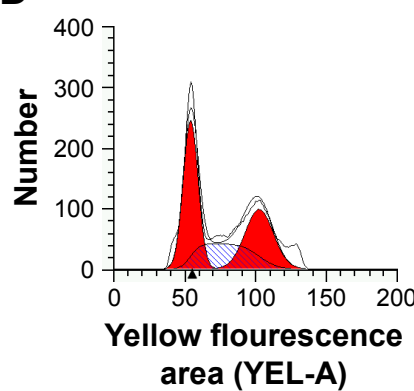

C

Con

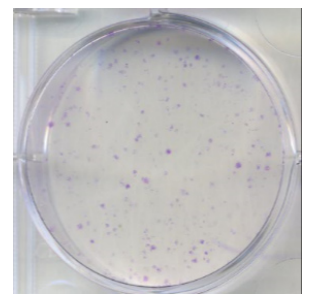

si-NC
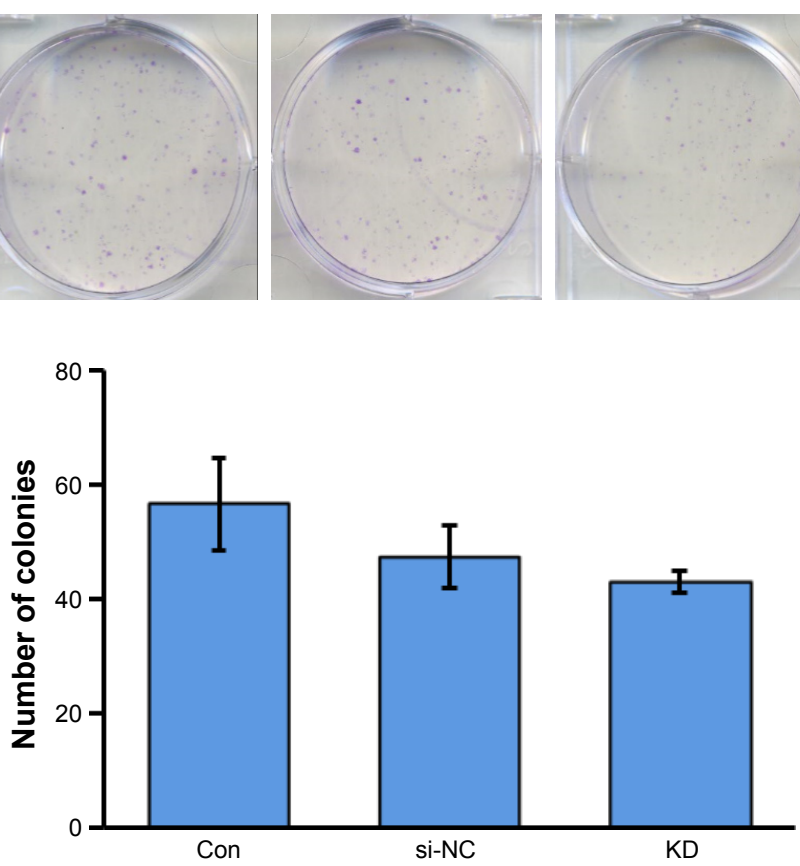

E
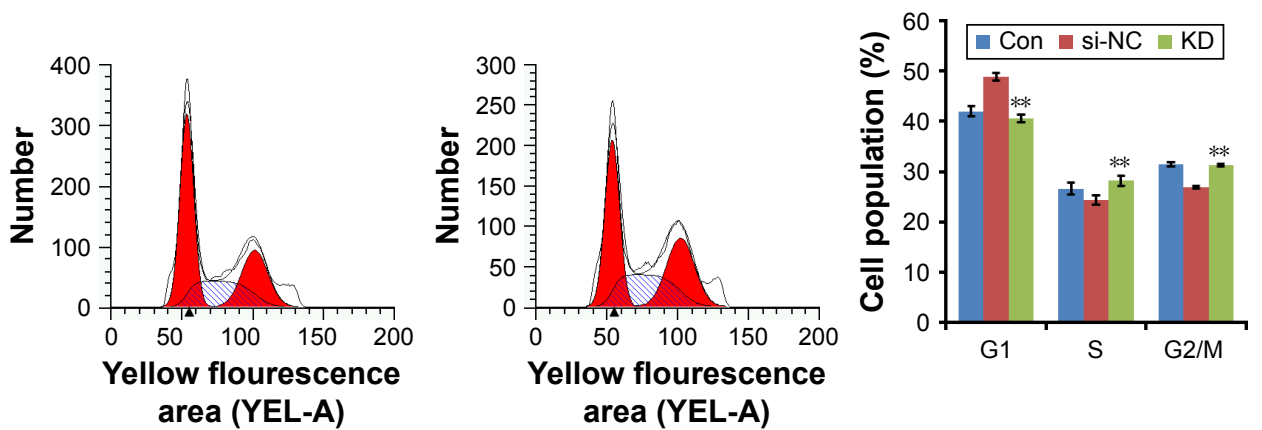

area (YEL-A)
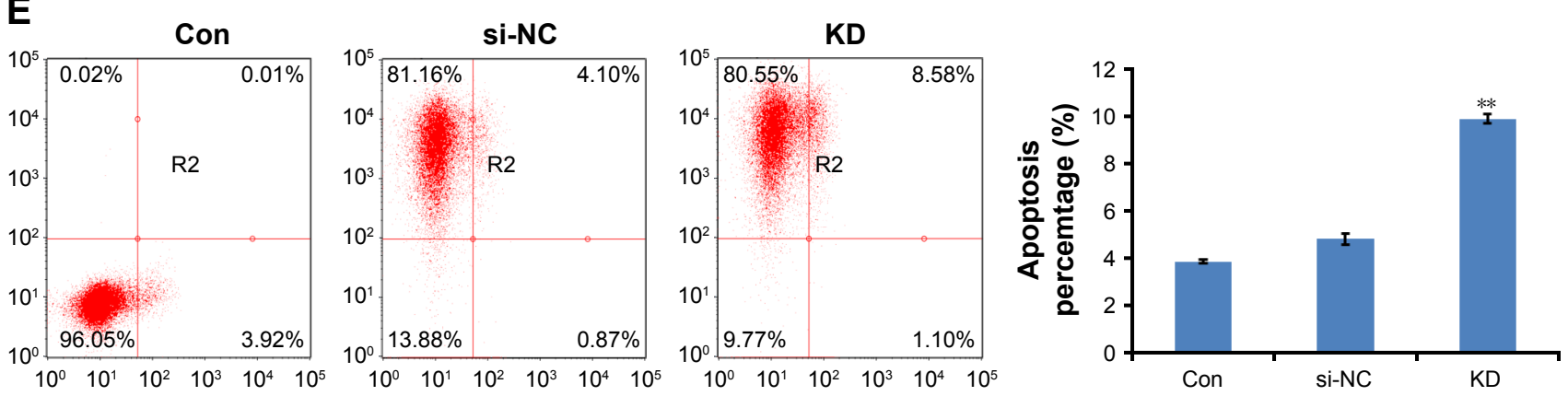

Figure 3 The effects of RPII-357HI4.17 on cell viability.

Notes: (A) The relative expression level of RPII-357HI4.17 in MGC-803 cell line was significantly decreased by si-RPII-357HI4.I7, and compared with si-NC. (B) MTT assay showed down-regulation of RPII-357HI4.I7 inhibited cell proliferation of MGC-803 cell line. (C) Colony-formation assays showed that down-regulation of RPII357HI4.17 signifiantly decreased the colony-forming ability of MGC-803 cell line. (D) Cell cycle analysis determined that down-regulation of RPII-357HI4.I7 expression promoted GI phase arrest in MGC-803 cell line. (E) Flow cytometric analysis showed induced cell apoptosis after si-RPII-357HI4.I7 transfection. The experiments were performed in triplicate. $* P<0.05$ compared with si-NC, $* * * 0.01$ compared with si-NC.

Abbreviations: CON, MGC-803 cell line; si-NC, si-negative control/non-targeting shRNA; KD, si-RPII-357HI4.I7. 


\section{RPI I-357HI4.I7 downregulation inhibits cell migration and invasion in DGC}

To assess whether RP11-357H14.17 is involved in DGC cell migration and invasion, we evaluated cell migration and invasion by wound healing and transwell assays. As shown in Figure 4A, the migration ability of RP11-357H14.17 knockdown MGC-803 cells was significantly reduced compared with the negative control (NC) cells $(P<0.05)$. Furthermore, migration and invasion were also markedly repressed, as shown in transwell invasion assays ( $P<0.05$, Figure 4B).

\section{Discussion}

Multiple studies have revealed the emerging significance of lncRNAs in various human diseases, including cancer. ${ }^{21-24}$ LncRNAs play critical roles in tumor invasion and metastasis, by regulating tumor suppressor and oncogenetic pathways. ${ }^{25,26}$ The relationship between lncRNAs and tumors attracts increasing attention from oncologists. Recently, more evidence has emerged that abnormal lncRNAs might play critical roles in GC invasion and metastasis. ${ }^{27,28}$ For example, our previous study found that high lncRNA H19 levels contribute to the proliferation of GC cells. ${ }^{29}$ The IncRNA HOTAIR functions as a competing endogenous RNA to regulate HER2 expression by sponging miR-331-3p in GC. ${ }^{30}$ Accumulating evidence suggests that further lncRNA assessment would provide new insights that may help find more efficient biomarkers of GC for early diagnosis, effective therapy, and prognosis evaluation.

To the best of our knowledge, this is the first report to illustrate RP11-357H14.17 function in DGC. In the present study, we first selected the lncRNA RP11-357H14.17 from a total of 112 lncRNAs associated with DGC by high-throughput
A
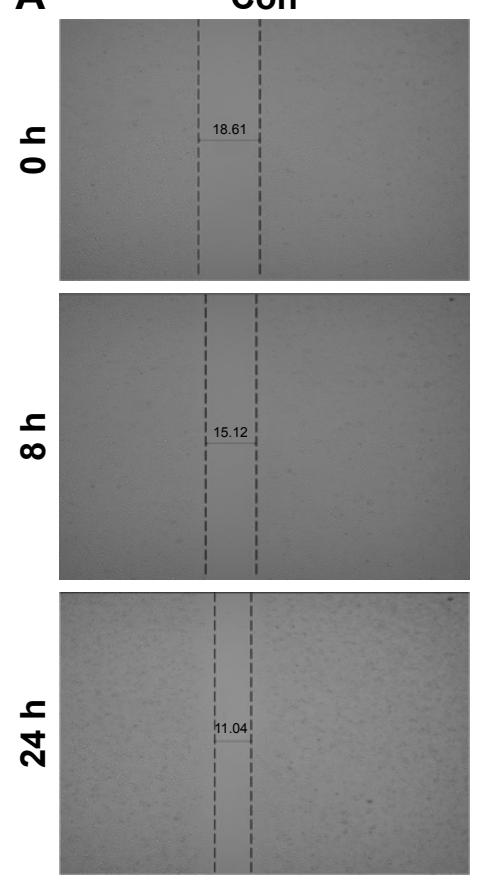

NC
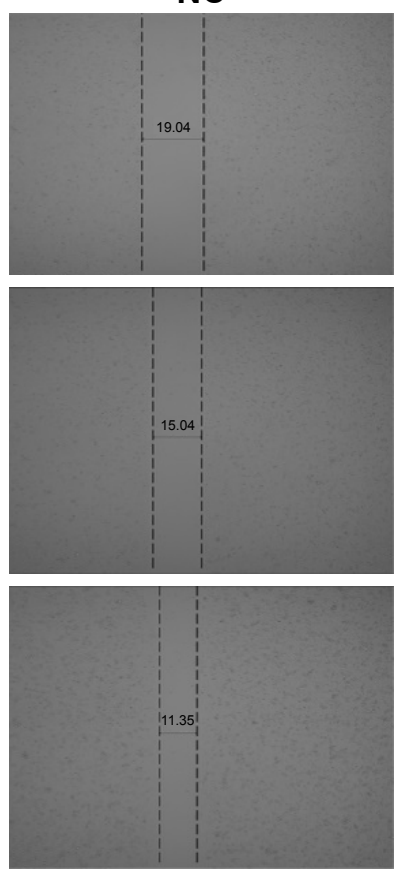

KD
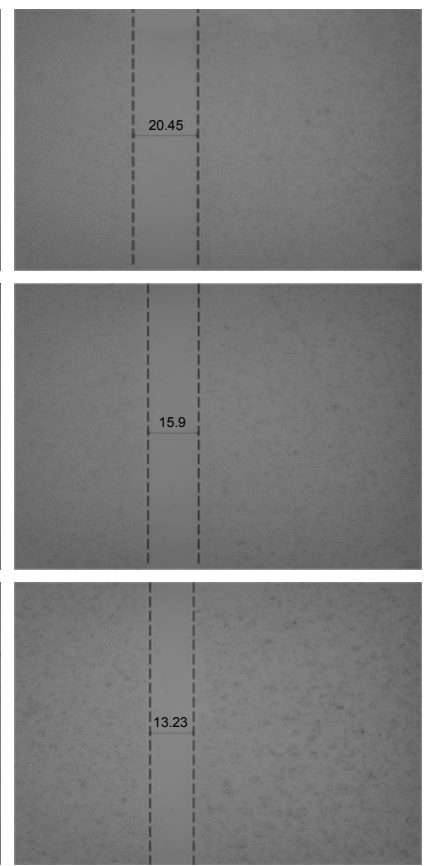

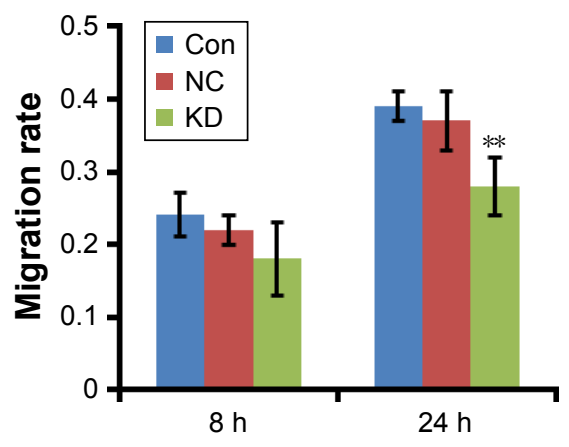

Figure 4 (Continued) 
B

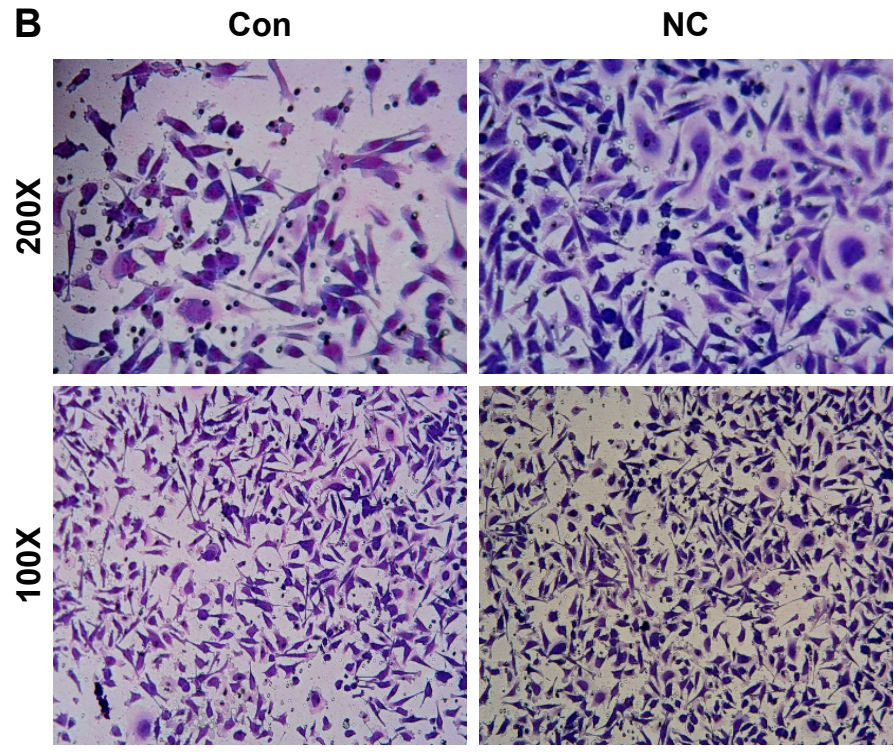

KD

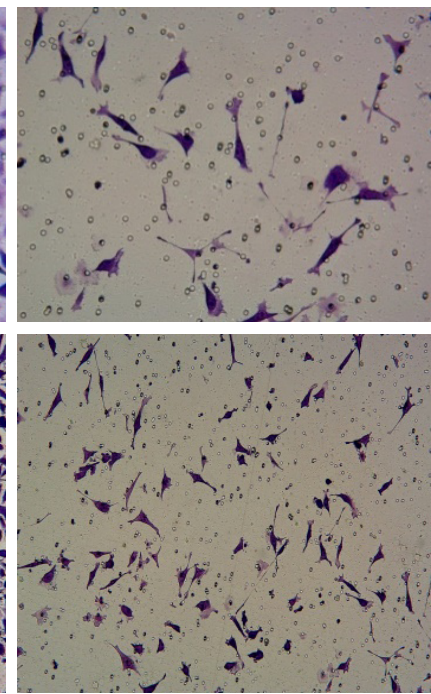

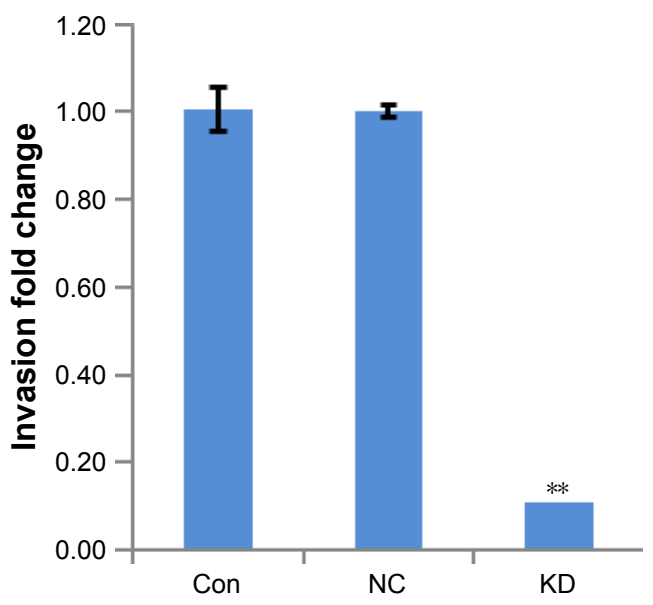

Figure 4 Knock-down RPII-357HI4.17 suppresses migration and invasion of DGC cells. Cell migration and invasion were determined by wound healing assay and transwell assay.

Notes: (A) Inhibition of migration of MGC-803 cell line by RPII-357HI4.I7 siRNA. (B) Inhibition of invasion of MGC-803 cell line by RPI-357HI4.I7 siRNA. Data are shown as mean \pm standard deviation. The experiments were all repeated at least three times. $* * P<0.01$ compared with $\mathrm{NC}$.

Abbreviations: DGC, diffuse-type gastric carcinoma; CON, MGC-803 cell line; NC, negative control; KD, si-RPII-357HI4.I7.

sequencing, and confirmed RP11-357H14.17 was highly expressed in DGC cells and tissues by RT-PCR. These findings may be the first evidence that high RP11-357H14.17 expression is closely associated with the development of DGC. Moreover, we evaluated the associations of RP11-357H14.17 expression levels with clinicopathological characteristics of DGC. Interestingly, high RP11-357H14.17 expression was more frequently correlated with aggressive tumor characteristics (greater invasion depth, higher tumor stage, lymphatic metastasis, and advanced tumor, node, metastasis (TNM) stage). This suggested that RP11-357H14.17 might be useful in the development of novel prognostic and progression markers in DGC.

Due to the abnormally high expression of RP11357H14.17 in DGC cells and tissues, we hypothesized that
RP11-357H14.17 plays a significant role in tumor biology. To examine this possibility, RP11-357H14.17 expression levels in four representative GC cell lines were evaluated comparatively with the normal gastric epithelial cell lines GES-1. As shown above, RP11-357H14.17 expression was significantly increased in the DGC cell line (MGC-803) compared with the normal gastric epithelial cell line GES-1, which corroborated our previous findings. We next used siRNA to knockdown RP11-357H14.17 in DGC cells; this inhibited cell proliferation, cell cycle progression, cell migration and cell invasion, and promoted apoptosis. These results revealed that RP11-357H14.17 might be involved in the progression of DGC by altering cell migration and invasion, and could constitute a potential prognostic biomarker and target for new antineoplastic therapies. 
The present study had some limitations. First, due to the short cycle of clinical specimens, survival analyses were not performed. Second, the effects of RP11-357H14.17 were evaluated in vitro, and in vivo effects of RP11-357H14.17 on DGC and the related mechanism were not analyzed in this study.

\section{Conclusion}

In summary, this study first confirmed that RP11-357H14.17 is significantly increased in DGC tissues and cell lines, with high RP11-357H14.17 expression correlated with invasion depth, larger tumor size, lymphatic metastasis, and TNM stage in DGC patients. Regulation of RP11-357H14.17 expression affected proliferation, migration and invasion of DGC. These findings suggested that RP11-357H14.17 is a potential therapeutic target for highly aggressive and malignant DGCs in clinical practice.

\section{Acknowledgment}

This work was supported by the National Natural Science Foundation of China (Grant No 81172156 and 81372048).

\section{Disclosure}

The authors report no conflicts of interest in this work.

\section{References}

1. Siegel RL, Miller KD, Jemal A. Cancer statistics, 2016. CA Cancer J Clin. 2016;66(1):7-30.

2. Lauren $P$. The two histological main types of gastric carcinoma: diffuse and so-called intestinal-type carcinoma. An attempt at a histo-clinical classification. Acta Pathol Microbiol Scand. 1965;64:31-49.

3. Van Cutsem E, Sagaert X, Topal B, Haustermans K, Prenen H. Gastric cancer. Lancet. 2016;388(10060):2654-2664.

4. Jinawath N, Furukawa Y, Hasegawa S, et al. Comparison of gene-expression profiles between diffuse- and intestinal-type gastric cancers using a genome-wide cDNA microarray. Oncogene. 2004;23(40):6830-6844.

5. Ming SC. Cellular and molecular pathology of gastric carcinoma and precursor lesions: a critical review. Gastric Cancer. 1998;1(1): 31-50.

6. Sipponen P. Gastric cancer: pathogenesis, risks, and prevention. J Gastroenterol. 2002;37(Suppl 13):39-44.

7. Hirose S, Honjou H, Nakagawa H, et al. Scirrhous carcinoma of the stomach: a clinical and pathological study of 106 surgical cases. Gastroenterol Jpn. 1989;24(5):481-487.

8. Otsuji E, Kuriu Y, Okamoto K, et al. Outcome of surgical treatment for patients with scirrhous carcinoma of the stomach. Am J Surg. 2004; 188(3):327-332.

OncoTargets and Therapy

\section{Publish your work in this journal}

OncoTargets and Therapy is an international, peer-reviewed, open access journal focusing on the pathological basis of all cancers, potential targets for therapy and treatment protocols employed to improve the management of cancer patients. The journal also focuses on the impact of management programs and new therapeutic agents and protocols on
9. Marques-Lespier JM, Gonzalez-Pons M, Cruz-Correa M. Current perspectives on gastric cancer. Gastroenterol Clin North Am. 2016; 45(3):413-428.

10. Correa P, Houghton J. Carcinogenesis of Helicobacter pylori. Gastroenterology. 2007;133(2):659-672.

11. Adachi Y, Yasuda K, Inomata M, Sato K, Shiraishi N, Kitano S. Pathology and prognosis of gastric carcinoma: well versus poorly differentiated type. Cancer. 2000;89(7):1418-1424.

12. Tahara E. Molecular biology of gastric cancer. World J Surg. 1995; 19(4):484-488; discussion 489-490.

13. Nagano T, Fraser P. No-nonsense functions for long noncoding RNAs. Cell. 2011;145(2):178-181.

14. Muers M. RNA: Genome-wide views of long non-coding RNAs. Nat Rev Genet. 2011;12(11):742.

15. Ponting CP, Oliver PL, Reik W. Evolution and functions of long noncoding RNAs. Cell. 2009;136(4):629-641.

16. Loewer S, Cabili MN, Guttman M, et al. Large intergenic non-coding RNA-RoR modulates reprogramming of human induced pluripotent stem cells. Nat Genet. 2010;42(12):1113-1117.

17. $\mathrm{LiH}, \mathrm{YuB}, \mathrm{LiJ}$, etal. Overexpression of IncRNAH19 enhances carcinogenesis and metastasis of gastric cancer. Oncotarget. 2014;5(8):2318-2329.

18. Zhang EB, Kong R, Yin DD, et al. Long noncoding RNA ANRIL indicates a poor prognosis of gastric cancer and promotes tumor growth by epigenetically silencing of miR-99a/miR-449a. Oncotarget. 2014;5(8):2276-2292.

19. Ma L, Zhou Y, Luo X, Gao H, Deng X, Jiang Y. Long non-coding RNA XIST promotes cell growth and invasion through regulating miR-497/ MACC1 axis in gastric cancer. Oncotarget. 2017;8(3):4125-4135.

20. Yuan CL, Li H, Zhu L, Liu Z, Zhou J, Shu Y. Aberrant expression of long noncoding RNA PVT1 and its diagnostic and prognostic significance in patients with gastric cancer. Neoplasma. 2016;63(3):442-449.

21. Gibb EA, Brown CJ, Lam WL. The functional role of long non-coding RNA in human carcinomas. Mol Cancer. 2011;10:38.

22. Ulitsky I, Bartel DP. lincRNAs: genomics, evolution, and mechanisms. Cell. 2013;154(1):26-46.

23. Geisler S, Coller J. RNA in unexpected places: long non-coding RNA functions in diverse cellular contexts. Nat Rev Mol Cell Bio. 2013; 14(11):699-712.

24. Wapinski O, Chang HY. Long noncoding RNAs and human disease. Trends Cell Biol. 2011;21(6):354-361.

25. Guttman M, Amit I, Garber M, et al. Chromatin signature reveals over a thousand highly conserved large non-coding RNAs in mammals. Nature. 2009;458(7235):223-227.

26. Huarte M, Guttman M, Feldser D, et al. A Large intergenicnoncoding RNA induced by $\mathrm{p} 53$ mediates global gene repression in the p53 response. Cell. 2010;142(3):409-419.

27. Fang XY, Pan HF, Leng RX, Ye DQ. Long noncoding RNAs: novel insights into gastric cancer. Cancer Lett. 2015;356(2):357-366.

28. Zhou X, Yin C, Dang Y, Ye F, Zhang G. Identification of the long non-coding RNA H19 in plasma as a novel biomarker for diagnosis of gastric cancer. Sci Rep. 2015;5:11516.

29. Yang F, Bi J, Xue X, et al. Up-regulated long non-coding RNA H19 contributes to proliferation of gastric cancer cells. FEBS J. 2012; 279(17):3159-3165.

30. Liu XH, Sun M, Nie FQ, et al. Lnc RNA HOTAIR functions as a competing endogenous RNA to regulate HER 2 expression by sponging miR-331-3p in gastric cancer. Mol Cancer. 2014;13:92.

\section{Dovepress}

patient perspectives such as quality of life, adherence and satisfaction. The manuscript management system is completely online and includes a very quick and fair peer-review system, which is all easy to use. Visit http://www.dovepress.com/testimonials.php to read real quotes from published authors. 